\title{
The impact of experimental diabetes mellitus in rats on glomerular capillary number and sizes
}

\author{
J. R. Nyengaard ${ }^{1}$ and R. Rasch $^{2}$ \\ ${ }^{1}$ Stereological Research Laboratory, University Institute of Pathology and Second University Clinic of Internal Medicine, \\ Institute of Experimental Clinical Research, \\ ${ }^{2}$ Department of Cell Biology, Institute of Anatomy, University of Aarhus, Denmark
}

\begin{abstract}
Summary. The structural counterpart of the increased glomerular filtration found in acute diabetes mellitus and experimental diabetes has been ascribed to the increased glomerular filtration surface. Using modern design-based stereological methods and light microscopy on perfusionfixed rat kidneys the average total surface area of capillaries per glomerulus in control rats was $291 \pm 4210^{-3} \mathrm{~mm}^{2}( \pm \mathrm{SD})$ increasing to $383 \pm 5510^{-3} \mathrm{~mm}^{2}$ in 10 -day-diabetic rats. There was a further increase to $469 \pm 7010^{-3} \mathrm{~mm}^{2}$ in 50 -day-diabetic rats. The average total length of capillaries per glomerulus increased from $12.5 \pm 2.2 \mathrm{~mm}$ in the control rats to $16.9 \pm$ $2.4 \mathrm{~mm}$ in the 10-day-diabetic rats whereas the further increase to $19.4 \pm 3.0 \mathrm{~mm}$ in the 50 -day-diabetic rats failed to reach statistical significance. The average number of topologically defined capillaries per glomerulus increased from
\end{abstract}

$215 \pm 29$ in the control rats to $260 \pm 45$ and $316 \pm 29$ in the 10-day-diabetic and 50-day-diabetic rats, respectively. The results showed just a slight increase in the mean length of a capillary from $58.1 \pm 6.2 \mu \mathrm{m}$ in the control rats to $65.6 \pm$ $2.6 \mu \mathrm{m}$ in the 10-day-diabetic rats after which it normalized in the 50-day-diabetic rats to $61.3 \pm 3.6 \mu \mathrm{m}$. The geometric factor or "resistance" in Poiseuille's law did not differ between the three groups, neither did the diameter of the capillaries, nor the number of glomeruli. The implications of the formation of capillaries in renal glomeruli are discussed and it is concluded that glomerular capillaries in experimental diabetes grow mainly by the formation of new capillaries.

Key words: Capillaries, experimental diabetes mellitus, glomerulus, kidney, rats, stereology, topology.
It is well-established that kidney volume and glomerular function increase in early diabetes mellitus [1] and in acute experimental diabetes [2]. The structural counterpart of the increased glomerular function has been ascribed to the increased glomerular filtration surface area as found both in human subjects with recent onset diabetes [3] as well as in rats with 4 days of streptozotocin diabetes [4]. The increased glomerular filtration surface area found in the 4-day-diabetic rats was mainly caused by an increased glomerular capillary length. This result raised the question as to whether such structural changes were due to an increase in length of the existing capillaries or due to the formation of new capillaries. A topological definition of a capillary unit [5] may help to answer this question by quantitating the three-dimensional changes of capillary number in the complex glomerular capillary network. The aim of this report was therefore to use design-based stereological methods for estimating the number, length, surface area and derived quantities of the glomerular capillaries in rats with short-term streptozotocin diabetes.

\section{Materials and methods}

\section{Animals}

Female Wistar rats (Møllegaards Avlslab., Eiby, Denmark) were weighed and randomly allocated into three groups before the experiment. The 10-day-diabetic rats (D10) were started 40 days later than the 50-day-diabetic rats (D50) so that all three groups of rats were the same age at the end of the experiment. The data of the three groups of rats including the control rats (C) are shown in Table 1. The animals had free access to standard rat chow (Altromin, Lage, FRG) throughout the experiment.

Diabetes was induced by a single i. p. injection of $90 \mathrm{mg}$ streptozotocin (Upjohn Inc., Kalamazoo, Ill, USA) per kilogram of body weight. Diabetes developed on the second day after streptozotocin administration, as verified by glucosuria and blood glucose measurements. The diabetic animals were injected daily with small doses of insulin (long acting heat treated Ultralente, ox, $\mathrm{pH} 5.5$, not for human use; Novo, Bagsværd, DK) [6]. Blood glucose was measured in the diabetic animals once a week using a BG-test-BG (Boehringer Mannheim, Mannheim, FRG) and Hypocount B (Hypoguard Ltd., Woodbridge, UK). Urine was tested for glucose with Diastix (Ames, Slough, UK). 
The rats were anaesthetised with pentobarbital $(50 \mathrm{mg} / \mathrm{kg})$ and their left kidneys were subjected to retrograde perfusion through the aorta [7]. The solution containing $1 \%$ glutaraldehyde and $3 \%$ paraformaldehyde in phosphate buffer was infused under a constant pressure of $18.7 \mathrm{kPa}$ for $5 \mathrm{~min}$. Only kidneys which blanched immediately were included. After perfusion the kidneys were weighed and they were all coded in order to evaluate them blindly.

\section{Tissue preparations}

A tissue slicer [8] was used for cutting the entire kidney into slices with a thickness of $1.5 \mathrm{~mm}$. Every third slice was sampled systematically random making three uniform sets of kidney slices. Three blocks were sampled uniformly from the cortex of the first set of tissue slices using a biopsy needle with a diameter of $1.5 \mathrm{~mm}$ and a plastic disc perforated with equidistantly spaced holes. The three tissue blocks were en bloc stained with uranyl acetate and embedded in Epon. The isector [9] ensured isotropic, uniform random sections. Three consecutive sections of $1.5 \mu \mathrm{m}$ thickness were cut from each Epon block using an LKB Historange microtome (Cambridge Instruments Ltd., Cambridge, UK) and the Epon sections were stained with toluidine blue. All estimations on the Epon sections were evaluated as a ratio of weighted sums on blocks. The weights were half the number of glomerular profiles in the middle section.

Another complete set of slices from each kidney was embedded together in a single capsule using glycolmethacrylate (Historesin, Cambridge Instruments). The plastic block was entirely sectioned into $20 \mu \mathrm{m}$ thick sections. From these $20 \mu \mathrm{m}$ thick sections every sixth section (the sampling section) was studied together with the next section, named the look-up section. One time in this section series, ten $2 \mu \mathrm{m}$ thick sections were cut instead of a single $20 \mu \mathrm{m}$ thick section. One of these thin sections per block was sampled. All sections were stained with periodic acid Schiff.

\section{Stereological techniques}

Glomerular number and size two identical Olympus BHS projection microscopes (Bico A/S, Glostrup, DK) equipped with 100-W halogen light sources and projection attachments $[10]$ were used to estimate the total number of glomeruli in the kidney, N(glom), with the fractionator [11]. One of the microscopes had a motor-driven microscope stage mounted for systematically random sampling a known fraction of fields of vision in the series of $20 \mu \mathrm{m}$ thick glycolmethacrylate sections. The glomeruli were counted if they disappeared from the sampling section to the look-up section and vice versa, $\mathrm{\Sigma Q}^{-}$, in the afore-mentioned fraction of the kidney. The total number of glomeruli is then the number of counted glomeruli divided by the total sampling fraction. The total sampling fraction comprises the fraction of sampled tissue slices, $\frac{1}{3}$, the fraction of sampled thick sections, $\frac{1}{6}$, the fraction of fields of vision, $\frac{1}{12}$, and the fraction related to the fact that some tissue was excluded for glomerular-counting and that glomeruli were counted both ways in the disector, $\frac{1}{1.1}$,

$\mathrm{N}($ glom $)=\Sigma \mathrm{Q}^{-} \cdot(\text { total sampling fraction })^{-1}$

Using a combination of the above-mentioned fractionator-sampling of glomeruli on the thick glycolmethacrylate sections, and pointcounting of glomeruli on the thin glycolmethacrylate sections the mean glomerular size, $\bar{v}$ (glom), was estimated. The section thickness $t$ of $20 \mu \mathrm{m}$ refers to the thick glycolmethacrylate sections. A point in the counting frame representing a certain area of the section, (frame area), was used for sampling a fraction of cortex systematically at random during glomerular counting, $\Sigma \mathrm{P}_{\mathrm{f}} . \Sigma \mathrm{P}$ (glom) was the number of points hitting glomeruli and $\Sigma \mathrm{P}$ (cor) was the number of points hitting cortex when moving the microscope stage with the thin glycolmethacrylate sections systematically at random. The mean glomerular size:

$$
\bar{v}(\text { glom })=\frac{V_{\mathrm{V}}}{N_{\mathrm{V}}}=\frac{\frac{\Sigma \mathrm{P}(\text { glom })}{\Sigma \mathrm{P}(\text { cor })}}{\frac{\Sigma \mathrm{Q}^{-}}{(\text {frame area }) \cdot t \cdot \Sigma \mathrm{P}_{\mathrm{f}}}}
$$

Cortex was here defined as the volume of the kidney superficial to the arcuate arteries [12]. The size and number estimation of glomeruli was recently described in detail [13] and was performed at a magnification of $165 \times$.

Estimation of number of glomerular capillaries. Using a low magnification of $165 \times$, two glomerular profiles were sampled on the middle one of the three consecutive Epon sections. The glomerular profiles should be located away from the edge at least one radius of the largest glomerular profiles to prevent sampling of incomplete and useless glomerular profiles at the edge [14]. At a magnification of 1670 using a $100 \times$ oil immersion objective, the area of the sampled glomerular profile, a(glo), enclosed in a minimal string polygon [4] was estimated by point-counting. The sampled glomerular capillaries from a complete glomerular profile were compared with the corresponding glomerular capillaries in the two neighbouring sections for estimating the contribution to the Euler-Poincare' characteristic, $\chi$ (Fig. 1). The two above-mentioned projection microscopes were used as a physical disector [15]. The middle section was used both as the sampled and the look-up section in relation to its two neighbouring sections. The numerical capillary density per glomerulus, $W_{\mathrm{V}}(\mathrm{cap} / \mathrm{glo})$ :

$\mathrm{W}_{\mathrm{V}}(\mathrm{cap} / \mathrm{glo})=\frac{-\Sigma \chi(\mathrm{cap})}{2 \cdot t \cdot \Sigma \mathrm{a}(\mathrm{glo})}$

$\mathrm{t}$ denoting thickness of the Epon Sections $(=1.5 \mu \mathrm{m})$. The average number of capillaries per glomerulus, $\overline{\mathrm{w}}(\mathrm{cap}, \mathrm{glo})$, is then

$\overline{\mathrm{w}}(\mathrm{cap}, \mathrm{glo})=\left(\overline{\mathrm{v}}(\mathrm{glo}) \cdot \mathrm{W}_{\mathrm{v}}(\mathrm{cap} / \mathrm{glo})\right)+2$

The total number of glomerular capillaries per kidney, W(cap), derived from the multiplication of $\overline{\mathrm{w}}(\mathrm{cap}, \mathrm{glo})$ with $\mathrm{N}$ (glo). The practical application and variability of the capillary number estimator has been described thoroughly by Nyengaard and Marcussen [16].

Estimation of length and surface area density of capillaries. All measurements were performed on the sampled glomerular profiles on the isotropic, uniform random sections of Epon at a magnification of $1670 \times$. The length density of capillaries per glomerulus, where $Q_{A}$ denotes the number of capillary profiles in a sampled glomerular profile, was estimated as

$\mathrm{L}_{\mathrm{V}}(\mathrm{cap} / \mathrm{glo})=2 \cdot \mathrm{Q}_{\mathrm{A}}$

The estimation of the surface area density of the capillaries per glomerulus, $S_{\mathrm{V}}$ (cap/glo), came from

$\mathrm{S}_{\mathrm{V}}(\mathrm{cap} / \mathrm{glo})=2 \cdot \mathrm{I}_{\mathrm{L}}$

$I_{L}$ denoting intersections between the test lines of a counting grid and the capillary surface area.

Table 1. Body weight before and efter experiment, kidney weight, blood glucose (BG) concentration, and glucosuria for the control (C), 10-day-diabetic (D10), and 50-day-diabetic rats (D50)

\begin{tabular}{llll}
\hline & $\mathrm{C}$ & $\mathrm{D} 10$ & $\mathrm{D} 50$ \\
\hline $\begin{array}{l}\text { Number of animals } \\
\begin{array}{l}\text { Body weight before } \\
\text { experiment (g) }\end{array}\end{array}$ & $724 \pm 2$ & $123 \pm 6$ & $122 \pm 2$ \\
$\begin{array}{l}\text { Body weight after } \\
\text { experiment (g) }\end{array}$ & $249 \pm 8$ & $203 \pm 12$ & $196 \pm 13$ \\
$\begin{array}{l}\text { Kidney weight (g) } \\
\text { BG concentration }\end{array}$ & $1.01 \pm 0.08$ & $1.21 \pm 0.11$ & $1.42 \pm 0.10$ \\
$\quad$ (mmol/l) & $6.1 \pm 0.7$ & $16.4 \pm 1.9$ & $19.5 \pm 1.5$ \\
Glucosuria (\%) & Negative & $>2$ & $>2$ \\
\hline
\end{tabular}

Values are mean $\pm \mathrm{SD}$ 


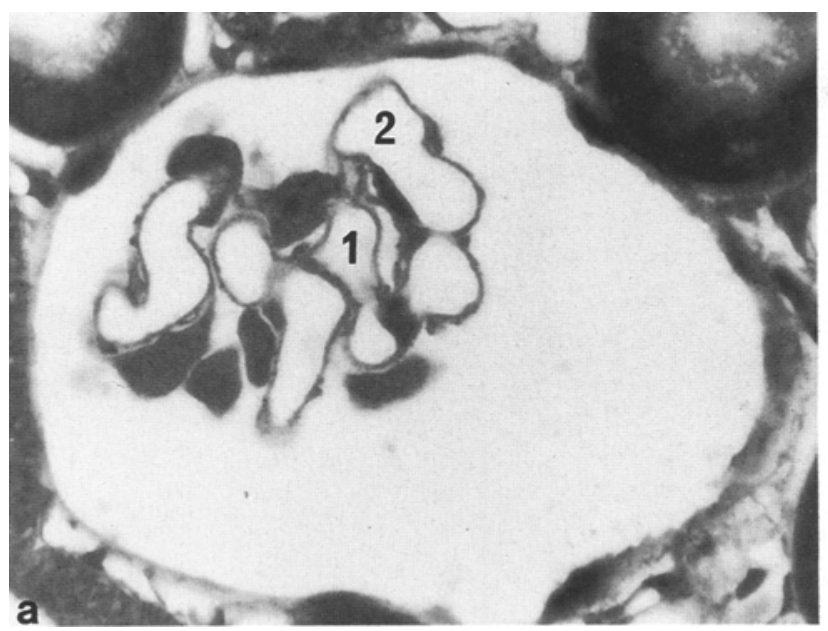

Fig. 1a,b. The contribution to the Euler number, $\chi$, is shown using two consecutive, toluidine blue stained Epon-sections from a rat glomerulus. It should be emphasized that the observer looks for the topological events of the capillary lumina. Capillary lumina 1 and 2 connect between section $\mathbf{a}$ and $\mathbf{b}$ and is still separate capillary lumina in both sections, thus giving rise to two "luminal connections". One capillary lumina, 3 , in section $\mathbf{a}$ is not seen in section $\mathbf{b}$ indicating one "luminal fragment". The rare "luminal lagoon", where an

Number, length, and, surface area of capillaries per glomerulus and of glomerular capillaries per kidney. The average quantities of total length, $\bar{L}$ (cap,glo), and total surface area, $\bar{S}($ cap,glo), of capillaries per glomerulus were obtained by multiplying the length and surface area densities with $\bar{v}$ (glo). When $\bar{L}$ (cap, glo), and S(cap,glo) were multiplied with $\mathrm{N}$ (glo), then the total length, L(cap), and surface area, S(cap), of glomerular capillaries per kidney were obtained, respectively.

Mean length, mean surface area, diameter, and cross-sectional area of capillaries. These values were all calculated from the above-mentioned estimates. Mean length of capillaries, $\overline{\mathrm{L}}$ (cap), was calculated from

$\overline{\mathrm{L}}($ cap $)=\frac{\overline{\mathrm{L}}(\text { cap,glo })}{\overline{\mathrm{w}}(\text { cap,glo })}$

mean surface area of capillaries, $\overline{\mathrm{s}}(\mathrm{cap})$, was obtained from

$\overline{\mathrm{s}}(\mathrm{cap})=\frac{\overline{\mathrm{S}}(\text { cap,glo })}{\overline{\mathrm{w}}(\text { cap,glo })}$

and assuming capillaries to be cylindrical tubes, the mean diameter of capillaries, $\bar{d}(\mathrm{cap})$, was derived from

$\overline{\mathrm{d}}(\mathrm{cap})=\frac{\overline{\mathrm{s}}(\mathrm{cap}, \mathrm{glo})}{\pi \cdot \overline{\mathrm{L}}(\mathrm{cap})}$

The mean cross-sectional area of capillaries, $\bar{a}($ cap) originated from

$\overrightarrow{\mathrm{a}}(\mathrm{cap})=\frac{\pi \cdot \overline{\mathrm{d}}^{2}(\mathrm{cap})}{4}$

Poiseuille's equation. The law of Poiseuille [17] relates a geometrical factor of the capillaries, L (cap) $/ \mathrm{a}^{2}$ (cap) comprising length, L (cap), and cross-sectional area, a(cap), of the capillary tube to the function of the capillaries, flow, $F$, and pressure drop, $\Delta \mathrm{P}$, via a constant. The constant is connected to the viscosity, $\eta$, of the fluid.

$\frac{\Delta \mathrm{P}}{\mathrm{F}}=8 \cdot \pi \cdot \eta \cdot \frac{\mathrm{L}(\mathrm{cap})}{\mathrm{a}^{2}(\mathrm{cap})}$

The very complicated network of parallel and serially connected capillaries does not allow sampling of the correct average of $\mathrm{L}$ (cap) $/ \mathrm{a}^{2}$ (cap), therefore this report approximated the original geo-

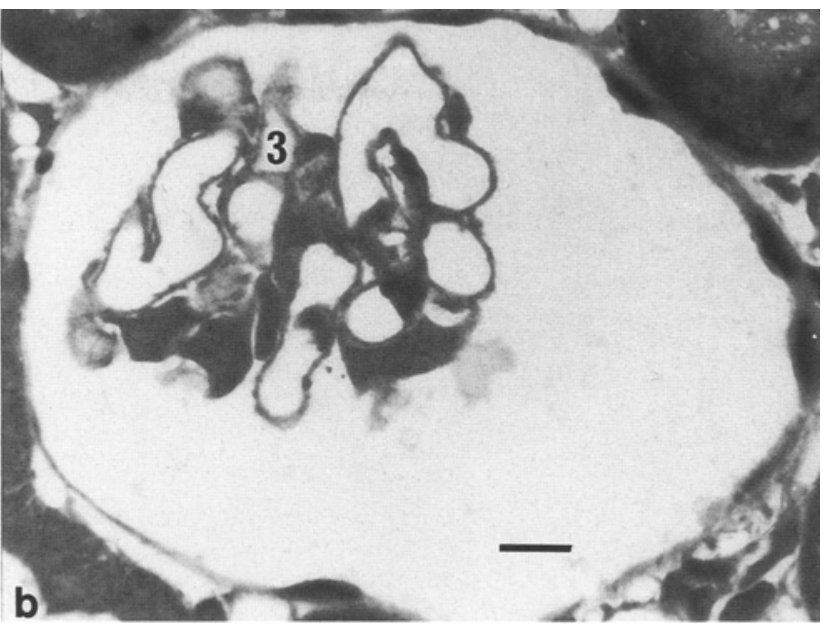

island of non-lumen appears inside the capillary lumen, is not seen here but is shown schematically in [16]. All topological events of the capillaries were evaluated in the disector [15]. The contribution to the Euler number;

$\Sigma \chi($ cap $)=\frac{1}{2}(\Sigma \#$ luminal fragments $+\Sigma$ \# luminal lagoons $-\Sigma \#$ luminal connections); $\Sigma \chi$ (cap) is used in Eq. 3 for estimating the capillary numerical density. The scale bar is $10 \mu \mathrm{m}$

metrical factor with $\overline{\mathrm{L}}$ (cap)/a $\overline{\mathrm{a}}^{2}$ (cap). Moreover, Poiseuille's law is strictly applicable only in tubes with fluids of constant viscosity and non-pulsatile, streamline flow. With all these assumptions taken into consideration, the law of Poiseuille provides an opportunity to relate the changes in geometry or "resistance" of glomerular capillaries to changes in the flow-pressure relationship in normal and diabetic rats.

\section{Statistical analysis}

The variation of the stereological tools at the level of blocks [18] equal to the coefficient of error $\left(\mathrm{CE}_{\text {ste }}\right)$ was estimated as the SEM divided by the arithmetic mean. $\mathrm{CE}_{\text {ste }}$ of the number of glomeruli was estimated using equations published previously [19]. The $\mathrm{CE}_{\text {ste }}$ of the capillary number, length, and surface area estimators have been estimated by formula 9 given previously [20]. The total variation of the above-mentioned estimates, $\mathrm{CV}_{\mathrm{tot}}=\frac{\mathrm{SD}}{\overline{\mathrm{x}}}$, comprises the biological, $\mathrm{CV}_{\text {bio }}$, and stereological variation: $\mathrm{CV}_{\mathrm{tot}}^{2}=\mathrm{CV}_{\text {bio }}^{2}+\mathrm{CE}_{\text {ste. }}^{2}$.

An unpaired, two-tailed $t$-test with a level of significance of 0.05 was used to test differences between groups.

\section{Results}

The estimates of $\mathrm{CV}_{\text {tot }}$ and $\mathrm{CE}_{\text {ste }}$ are shown in Table 2 . The number of glomeruli was $27.6 \pm 2.910^{3}( \pm \mathrm{SD}$ ) for the $\mathrm{C}$ rats, $27.0 \pm 2.910^{3}$ for the D10 rats, $28.0 \pm 3.210^{3}$ for the

Table 2. The coefficient of methodological error, $\mathrm{CE}_{\mathrm{ste}}$, and the coefficient of interanimal variation, $\mathrm{CV}_{\text {tot }}$, are shown for the stereological estimators of mean glomerular volume, $\bar{v}$ (glo), average total number of capillaries per glomerulus, $\bar{w}($ cap,glo), average total length of capillaries per glomerulus, $\overline{\mathrm{L}}(\mathrm{cap}, \mathrm{glo})$, and average total surface area of capillaries per glomerulus, $S$ (cap,glo)

\begin{tabular}{lllll}
\hline & $\overline{\mathrm{v}}($ glo $)$ & $\overline{\mathrm{w}}($ cap,glo $)$ & $\overline{\mathrm{L}}$ (cap,glo) & $\overline{\mathrm{S}}$ (cap,glo $)$ \\
\hline $\mathrm{CE}_{\text {ste }}$ & 0.05 & 0.13 & 0.09 & 0.07 \\
$\mathrm{CV}_{\text {tot }}$ & 0.11 & 0.15 & 0.16 & 0.15 \\
\hline
\end{tabular}



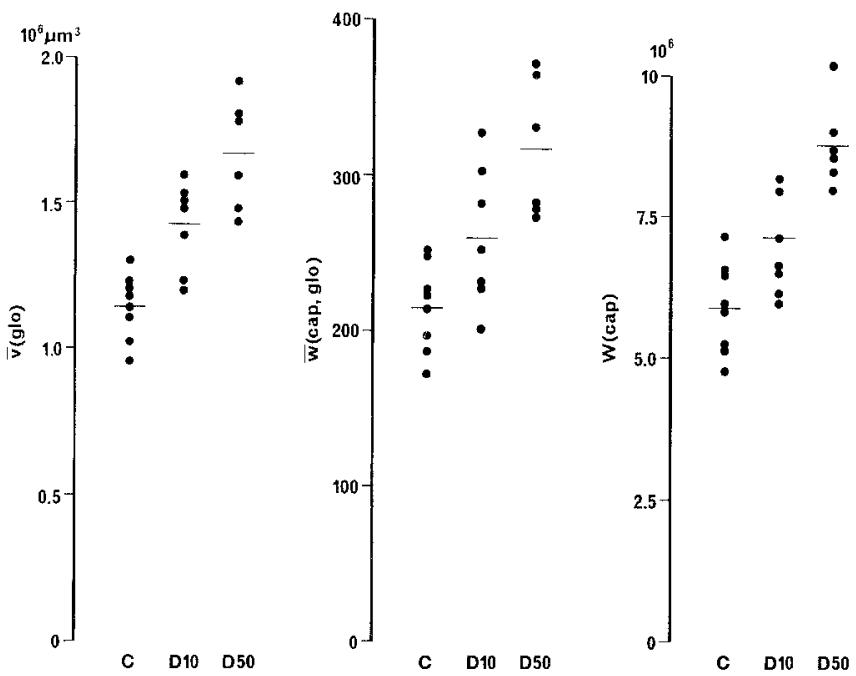

Fig. 2. The mean volume of glomeruli, $\bar{v}($ glo $)$, the average total number of capillaries per glomerulus, $\overline{\mathrm{w}}(\mathrm{cap}, \mathrm{glo})$, and total number of glomerular capillaries per kidney, W(cap), are shown for control rats (C), 10-day-diabetic rats (D10), and 50-day-diabetic rats (D50). The spacefilling of each capillary is $5300 \mu \mathrm{m}^{3}$ in the Crats, $5730 \mu \mathrm{m}^{3}$ in the D10 rats and $5280 \mu \mathrm{m}^{3}$ in the D50 rats
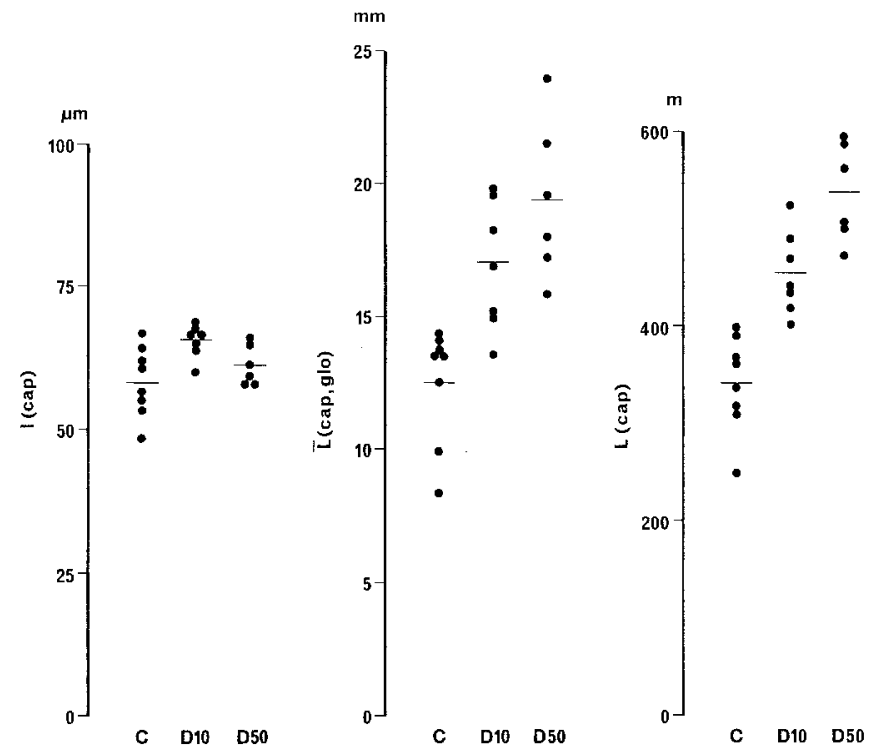

Fig. 3. The mean length of glomerular capillaries, $\bar{L}$ (cap), the average total length of capillaries per glomerulus, $\overline{\mathrm{L}}$ (cap,glo), and total length of glomerular capillaries per kidney, L(cap), are shown for control rats (C), 10-day-diabetic rats (D10), and 50-day-diabetic rats (D50)

D50 rats, and there was no significant difference between the three groups $(2 p>0.05)$. The mean volume of glomeruli increased from $1.14 \pm 0.1110^{6} \mu \mathrm{m}^{3}$ in the $\mathrm{C}$ rats to $1.49 \pm 0.1510^{6} \mu \mathrm{m}^{3}$ in the D10 rats $(2 p<0.05)$ and a further increase was seen in the D50 rats $1.67 \pm 0.1910^{6} \mu \mathrm{m}^{3}$ $(2 p<0.05)$ (Fig. 2). The average total number of capillaries per glomerulus increased from $215 \pm 29$ in the $C$ rats to $260 \pm 45$ in the D10 rats. There was a further increase to $316 \pm 45$ in the D50 rats $(2 p<0.05)$ (Fig. 2$)$. The total number of glomerular capillaries per kidney increased from $5.89 \pm 0.8310^{6}$ in the C rats to $6.94 \pm 0.8310^{6}$ in the D10 rats with a further increase to $8.77 \pm 0.7910^{6}$ in the D50

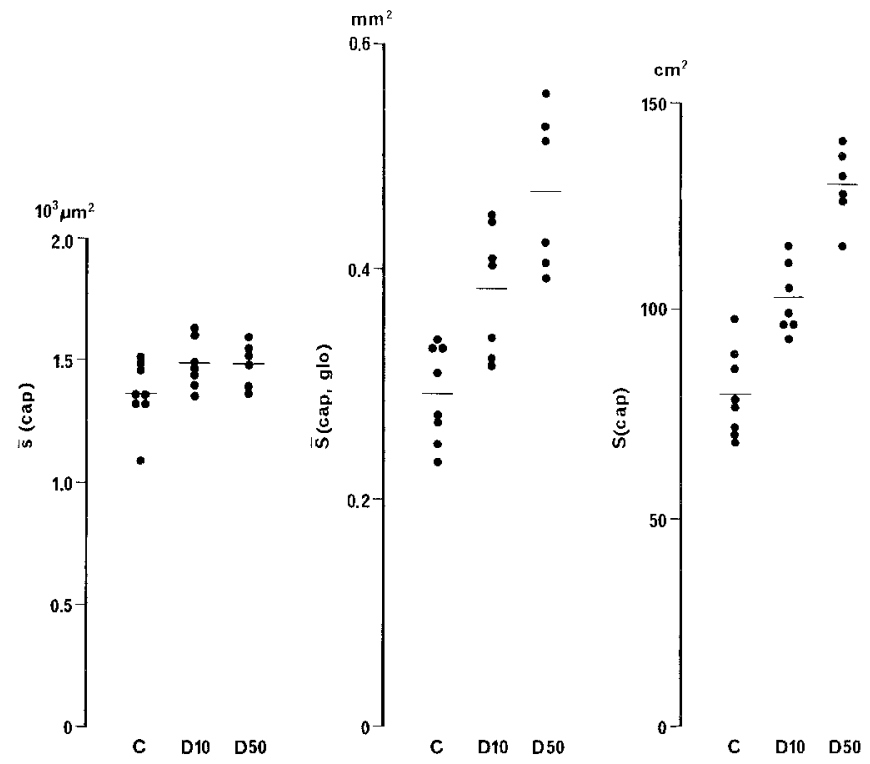

Fig.4. The mean surface area of glomerular capillaries, $\underline{\bar{s}}(\mathrm{cap})$, the average total surface area of capillaries per glomerulus, $\bar{S}($ cap,glo), and total surface area of glomerular capillaries per kidney, S(cap), are shown for control rats (C), 10-day-diabetic rats (D10), and 50 -day-diabetic rats (D50)

rats $(2 p<0.05)$ (Fig. 2). The average total length of capillaries per glomerulus was $12.5 \pm 2.2 \mathrm{~mm}$ in the $\mathrm{C}$ rats and increased to $16.9 \pm 2.4 \mathrm{~mm}(2 p>0.05)$ in the D10 rats whereas the increase to $19.4 \pm 3.0 \mathrm{~mm}$ in the D50 rats failed to reach statistical significance when compared with the D10 rats $(2 p=0.13)$ (Fig. 3). The total length of glomerular capillaries per kidney was $341 \pm 48 \mathrm{~m}$ in the $\mathrm{C}$ rats and increased to $453 \pm 42 \mathrm{~m}$ in the D10 rats with a further increase of $\mathrm{L}$ (cap) from the D10 to the D50 rats, $538 \pm 52 \mathrm{~m}(2 p<0.05)$ (Fig. 3). The average total surface area of capillaries per glomerulus increased from $291 \pm 42$ $10^{-3} \mathrm{~mm}^{2}$ in the $\mathrm{C}$ rats to $383 \pm 5510^{-3} \mathrm{~mm}^{2}$ in the D10 rats. There was a further increase to $469 \pm 7010^{-3} \mathrm{~mm}^{2}$ in the D50 rats $(2 p<0.05)$ (Fig. 4$)$. The total surface area of glomerular capillaries per kidney was $79.8 \pm 10.3 \mathrm{~cm}^{2}$ in the $C$ rats increasing to $102 \pm 8 \mathrm{~cm}^{2}$ in the D10 rats and with a further increase to $130 \pm 9 \mathrm{~cm}^{2}$ in the D50 rats $(2 p<0.05)$ (Fig. 4$)$.

The mean length of the topologically defined glomerular capillaries of $58.1 \pm 6.2 \mu \mathrm{m}$ in the $\mathrm{C}$ rats, increased to $65.6 \pm 2.6 \mu \mathrm{m}(2 p<0.05)$ in the D10 rats after which it normalized in the D50 rats to $61.3 \pm 3.6 \mu \mathrm{m}(2 p<0.05$ and $2 p>0.05$ D50 vs D10 and C rats, respectively) (Fig. 3). There was a tendency towards an increase in the mean surface area of glomerular capillaries from the $\mathrm{C}$ rats, $1.36 \pm 0.1310^{3} \mu^{2}$, to the D10 rats, $1.48 \pm 0.9910^{3} \mu \mathrm{m}^{2}$ $(2 p=0.07)$ and the D50 rats, $1.49 \pm 0.8310^{3} \mu \mathrm{m}^{2}(2 p=$ 0.07 ) (Fig.4). There was a significant increase in the diameter of the glomerular capillaries from the D10 rats, $7.20 \pm 0.33 \mu \mathrm{m}$ to the D50 rats, $7.72 \pm 0.51 \mu \mathrm{m}(2 p<0.05)$ but no significant difference between the $C$ rats, $7.50 \pm 0.82 \mu \mathrm{m}$, vs the D10 and D50 rats. Neither was there any difference in the geometrical factor or "resistance" from the Poiseuille-law between the C, $33.5 \pm 15.310^{-3}$ $\mu \mathrm{m}^{-3}$, and the D10 rats, $40.3 \pm 7.410^{-3} \mu \mathrm{m}^{-3}(2 p>0.05)$, 


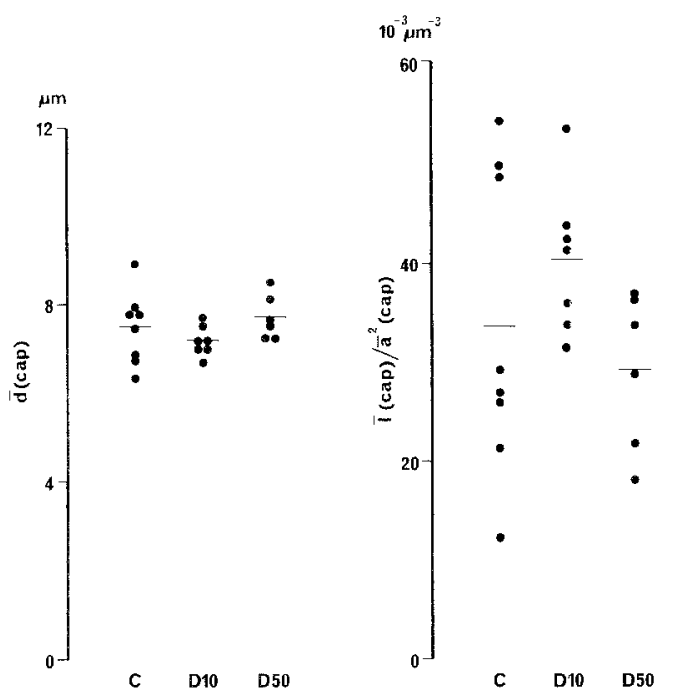

Fig.5. The mean diameter, $\overline{\mathrm{d}}(\mathrm{cap})$, and the "geometric factor" or resistance from the law of Poiseuille, $\bar{L}$ (cap) $/ \mathrm{a}^{2}$ (cap), are shown for control rats (C), 10-day-diabetic rats (D10), and 50-day-diabetic rats (D50)

nor was there any difference between the C and the D50 rats, $29.1 \pm 7.910^{-3}{\mu \mathrm{m}^{-3}}^{-2}(2 p>0.05)$. However, there was a decrease in "resistance" between the D10 to the D50 rats $(2 p<0.05)$ (Fig. 5$)$.

\section{Discussion}

This report has for the first time estimated the number of glomerular capillaries in rats with acute diabetes and shown that the number of capillaries in the complicated glomerular capillary network increases after induction of diabetes in rats. The results indicate that the increase of capillary loops in the glomerular capillary network is temporarily exceeded by the length-growth in the 10-daydiabetic rats whereas the mean length of glomerular capillaries is the same in the C and the D50 rats. The lengthening of glomerular capillaries may therefore be slightly more pronounced than the branching of glomerular capillaries in the first days of experimental diabetes. After weeks of experimental diabetes the branching seemingly balances the total length increase. The formation of a new capillary is quite a complicated process, particularly when comparing it to the simple lengthening of an existing capillary segment. This is the case, both when a new capillary starts from an existing capillary as a budding-out which fuses with another capillary in the vicinity [21], or when it is the result of intussusceptive growth [22]. When taking this into consideration, it is then surprising that the glomerular capillary network is able to make a lot of new capillary loops in acute experimental diabetes. Other studies of rats have found capillary proliferation in normal growing glomeruli [23] and in hypertrophied glomeruli induced by lithium-nephropathy [24] or nephrectomy [25], whereas the small atubular glomeruli in lithium-induced nephropathy had a smaller number of glomerular capillaries [24]. This might indicate that the number of glomerular capillaries is an important variable in glomerular atrophy or hypertrophy. One advantage of making all these new capillary loops instead of a simple lengthening of the existing capillary segments is that in the case of a blockade of a single capillary segment the blood can easily be directed to other segments [26].

The "resistance" of the glomerular capillaries as judged by the geometric factor in Poiseuille's law does not differ between the control and the diabetic rats, whereas the resistance falls from the D10 to the D50 rats. Østerby and Gundersen [4] approximated L(cap) from Poiseuille's law with total glomerular capillary length per kidney and found an increase in "resistance" of glomerular capillaries after 4 days of experimental diabetes and a decrease after 47 days. Their rats were older and not treated with insulin and it is striking that the total length of glomerular capillaries per kidney was unchanged after 47 days of experimental diabetes. However, it must be emphasized that the exact size of the resistance of the glomerular capillary network was neither estimated in this report nor in the previous study [4] because of lack of knowledge of the relationship between parallel and serially connected capillaries. We are as yet unable to obtain the exact resistance of glomerular capillaries, though it seems obvious that the formation of new capillaries, instead of lengthening the existing capillaries, other things being equal, must produce a network with a lower resistance.

Studies using light microscopic autoradiography with a labelling time of $1 \mathrm{~h}$ showed a lack of ${ }^{3} \mathrm{H}$-thymidine incorporation in glomeruli, in experimental diabetes of $2,4,6$ [27] and 50 days duration (R Rasch, personal communication). In contrast, glomerular cell number had increased in long-term diabetes mellitus [28]. We believe that glomerular endothelial cells may proliferate in experimental diabetes too, as a preliminary, light microscopic study might indicate: two control and two 50-day-diabetic rats had 247,203 and 319,241, glomerular endothelial cells, respectively (J.R. Nyengaard, personal communication).

Currently, we do not think that there is any connection between the increased glomerular capillary number found in diabetic rats and the diabetic glomerulopathy seen in diabetic subjects, mainly because an increased glomerular capillary number also can be found in other forms of glomerular hypertrophy in rats $[24,25]$. It is, however, interesting that isolated capillaries with a fluffy intrinsic structure associated with new vessels have been found in advanced stages of glomerulopathy in diabetic subjects [29]. It is therefore tempting to compare the formation of new glomerular capillaries in advanced diabetic nephropathy [29] with the conditions in diabetic retinopathy where capillary proliferation in retina is a well-known complication [30]. The occurrence of capillary formation may be understood as one phenomenon of generalized diabetic microangiopathy, but whether it is induced by the same factor is still a matter of debate. However, a fairly good relationship exists between urinary albumin excretion rate and diabetic retinopathy $[31,32]$.

In conclusion, this report has shown that rat glomerular capillaries in glomerular hypertrophy in experimental diabetes grow by formation of new capillaries rather than elongation of the existing capillaries - just as in other situations with glomerular hypertrophy. 
Acknowledgements. Professor H.J.G.Gundersen is gratefully thanked for help and discussions. The skillful technical assistance of A.M.Funder, L.L. Gjerrild and A.Larsen is thankfully acknowledged. The study was supported by the Danish Diabetes Association, the Danish Medical Research Council, Fonden af 17-12-1981, Fonden af 1870, Fonden til lægevidenskabens Fremme, Fru Ruth I.E. Kønig-Petersens forskningsfond, Kong Christian den Tiendes Fond, NOVO Foundation, Nyreforeningens Forskningsfond, and Aarhus University Research Foundation.

\section{References}

1. Mogensen CE, Andersen MJ (1973) Increased kidney size and glomerular filtration rate in early juvenile diabetes. Diabetes 22 : 706-712

2. Hostetter TH, Troy JL, Brenner BM (1981) Glomerular hemodynamics in experimental diabetes mellitus. Kidney Int 19:410415

3. Kroustrup JP, Gundersen HJG, Østerby R (1977) Glomerular size and structure in diabetes mellitus. III. Early enlargement of the capillary surface. Diabetologia 13: 207-210

4. Østerby R, Gundersen HJG (1980) Fast accumulation of basement membrane material and the rate of morphological changes in acute experimental diabetic glomerular hypertrophy. Diabetologia 18: 493-500

5. Nyengaard JR, Bendtsen TF, Gundersen HJG (1988) Stereological estimation of the number of capillaries, exemplified by renal glomeruli. APMIS 4: 92-99

6. Rasch R (1979) Control of blood glucose levels in streptozotocin diabetic rat using a long-term heat-treated insulin. Diabetologia $16: 185-190$

7. Maunsbach AB (1966) The influence of different fixatives and fixation methods on the ultrastructure of rat kidney proximal tubule cells. I. Comparison of different perfusion fixation methods and of glutaraldehyde, formaldehyde and osmium tetroxide fixatives. J Ultrastr Res 15: 242-282

8. Gundersen HJG, Jensen EB (1987) The efficiency of systematic sampling in stereology and its prediction. J Microsc 147: 229-263

9. Nyengaard JR, Gundersen HJG (1992) The isector: a simple and direct method for generating isotropic, uniform random sections from small specimens. J Microsc 165: 427-431

10. Sørensen FB (1991) Stereological estimation of the mean and variance of nuclear volume from vertical sections. J Microsc 162: 203-229

11. Gundersen HJG (1986) Stereology of arbitrary particles. A review of unbiased number and size estimators and the presentation of some new ones, in memory of William R. Thompson. J Microsc 143: 3-45

12. Kriz W, Bankir L (1988) A standard nomenclature for structures of the kidney. Kidney Int 33:1-7

13. Nyengaard JR, Bendtsen TF (1990) A practical method to count the number of glomeruli in the kidney as simplified in a range of animals. Acta Stereol 9:245-253

14. Østerby R, Gundersen HJG (1978) Sampling problems in the kidney. In: Miles RE, Serra J (eds) Lecture notes in biomathematics. Springer-Verlag, Berlin, pp 185-191

15. Sterio DC (1984) The unbiased estimation of number and sizes of arbitrary particles using the disector. J Microsc 134: 127-136

16. Nyengaard JR, Marcussen M (1992) The number of glomerular capillaries estimated by an unbiased and efficient method. $\mathbf{J}$ Microsc (In press)
17. White FN (1982) Circulation. In: Gordon MS, Bartholomew GA, Grinnel AD, Jørgensen CB, White FN (eds) Animal physiology. Principles and adaptations, Vol 4. Macmillan Publishing Co., New York, pp 197-271

18. Gundersen HJG, Østerby R (1980) Optimizing sampling efficiency of stereological studies in biology: or 'Do more less well'. J Microsc 121: 65-73

19. West MJ, Gundersen HJG (1990) Unbiased stereological estimation of the number of neurons in the human hippocampus. $J$ Comp Neurol 296: 1-22

20. Kroustrup JP, Gundersen HJG (1983) Sampling problems in an heterogeneous organ: quantitation of relative and total volume of pancreatic islets by light microscopy. J Microsc 132: 43-55

21. Naito I (1984) The development of glomerular capillary tufts of the bullfrog kidney from a straight interstitial vessel to an anastomosed capillary network. A scanning electron microscopic study of vascular casts. Arch Hist Jap 47: 441-456

22. Burri PH, Tarek MR (1990) A novel mechanism of capillary growth in the rat pulmonary microcirculation. Anat Rec 228: $35-45$

23. Nyengaard JR (1991) Unbiased estimation of capillary number in renal glomeruli from rats of varying age. Proc Eighth Int Congress for Stereology: 76 (Abstract)

24. Christensen S, Marcussen N (1992) Glomerular volume and the number of glomerular capillaries in lithium nephropathy in rats. Acta Physiol Scand 608: [Suppl] 185 (Abstract)

25. Nyengaard JR (1992) The number and dimensions of rat glomerular capillaries during normal development and after neonatal and adult nephrectomy. Kidney Int (In press)

26. Steinhausen M, Endlich K, Wiegman DL (1990) Glomerular blood flow. Kidney Int 38: 769-784

27. Rasch R, Nørgaard JOR (1983) Renal enlargement: comparative autoradiographic studies of ${ }^{3} \mathrm{H}$-thymidine uptake in diabetic and uninephrectomized rats. Diabetologia 25: 280-287

28. Gundersen HJG, Østerby R (1977) Glomerular size and structure in diabetes mellitus. II. Late abnormalities. Diabetologia 13: 43-48

29. Østerby R, Nyberg G (1987) New vessel formation in the renal corpuscles in advanced diabetic glomerulopathy. J Diabetic Compl 1: 122-127

30. Lundbæk K (1953) Long-term diabetes. Munksgaard, Copenhagen.

31. Kostraba JN, Klein R, Dorman JS et al. (1991) The epidemiology of diabetes complications study. IV. Correlates of diabetic background and proliferative retinopathy. Am J Epidemiol 133: 381 391

32. Vigstrup J, Mogensen CE (1985) Proliferative diabetic retinopathy: at risk patients identified by early detection of microalbuminuria. Acta Ophthalmol 63: 530-534

Received: 22 July 1992

and in revised form: 20 October 1992

Dr. J.R. Nyengaard

Stereological Research Laboratory

Bartholin Building

Aarhus University

DK-8000 Aarhus C

Denmark 